\title{
Footplate Incision in Rhinoplasty: an Update
}

\section{Jae-A Jung, Sik Namgoong, Seung-Kyu Han, Seong-Ho Jeong, Eun-Sang Dhong, Woo-Kyung Kim}

Department of Plastic Surgery, Korea University Guro Hospital, Korea University College of Medicine, Seoul, Korea

No potential conflict of interest relevant to this article was reported.
Background In 2004, we reported on 110 consecutive Asian rhinoplasty patients who were treated with the addition of a footplate incision to obtain a greater aesthetic satisfaction. We continue to perform the reported technique with several modifications, and we still think that this method contributes to Asian rhinoplasty.

Methods A footplate incision was made along the caudal border of the footplate of the medial crura onto the floor of the nasal vestibule. This incision can be made alone or in combination with either endonasal or open rhinoplasty. It enables surgeons to achieve a further tip projection since the pressure of the skin flap is reduced on the tip. In this study, we emphasize a couple of recent changes that we made to our procedure after publication of the prior article. First, we excised the dog-ear that appeared at the caudal end of the extended footplate incision. After making the new tip, a dog ear can be seen at the posterior end of the footplate incision. Second, an inferior columellar dissection was also extended to achieve an additional tip projection and to improve the columello-labial angle.

Results This study included 85 consecutive patients who underwent an aesthetic rhinoplasty using footplate incision techniques between August of 2010 and May of 2013. A total of 43 patients had an adequate follow-up time of over 12 months. The majority of the patients ( $40 / 43$ cases) were satisfied with the results.

Conclusions The authors believe that a footplate incision in aesthetic rhinoplasty is safe and can reliably achieve better results for Asian patients.

Keyword Rhinoplasty

\section{INTRODUCTION}

Aesthetic rhinoplasty procedures have increased in frequency in the Asian population. Although aesthetic concepts differ for each race, the current concept of nasal beauty shows a tendency to coincide with that of Caucasians. The ideal, beautiful nose tends to have a straight dorsum from the radix to the tip, with an adequate height. The tip should be slightly more prominent than the dorsum, and the columella must be of sufficient length to provide the necessary

Received: Jun 6, 2015 Revised: Jun 23, 2015 Accepted: Jun 23, 2015 Correspondence: Seung-Kyu Han Department of Plastic Surgery, Korea University Guro Hospital, 148 Gurodong-ro, Guro-gu, Seoul 152-703, Korea. E-mail: pshan@kumc.or.kr

Copyright @ 2015 The Korean Society for Aesthetic Plastic Surgery.

This is an Open Access article distributed under the terms of the Creative Commons Attribution Non-Commercial License (http://creativecommons.org/licenses/by-nc/3.0/) which permits unrestricted non-commercial use, distribution, and reproduction in any medium, provided the original work is properly cited. $\quad$ www.e-aaps.org projection of the nasal tip above the dorsal line. The nasolabial angle should be between 90 and 110 degrees, and elliptical pear-shaped nostrils are preferred. Asians commonly desire aesthetic rhinoplasty because of a flat or low dorsum, acute nasolabial angle, poorly projected and caudally rotated tip, short columella, wide alar base, and a round or flared nostril shape [1-3]. Therefore, the surgical procedure mainly focuses on dorsal augmentation and tip projection [4]. The low nasion and dorsum can be augmented with various methods using autogenous or alloplastic materials. However, a bulbous nasal tip, wide alar base, acute nasolabial angle, and recessive pyriform fossae further compound the difficulties. The surgeon can manipulate the footplate area of the medial crus (which plays an important role in nasal tip projection, nasolabial angle, length of the columella, and nostril shape) to achieve the desired effect. In Asians, the length of the footplate segment is much longer than in Caucasians, and since Caucasians and Asians have anatomic differences in their nasal configurations, a different surgical approach would be needed for each of the different races. 
In 2004, we reported on 110 consecutive Asian patients who underwent aesthetic rhinoplasty with the addition of a footplate incision to obtain greater satisfaction [5]. Nowadays we still perform the reported technique with several modifications. During the long term follow-up since publication of the article, no significant complications were reported and we still think that this method contributes to Asian Rhinoplasty. In this follow-up study, we report on our new cases and focus on a few changes.

\section{METHODS}

All procedures were performed under local anesthetic infiltration (1\% lidocaine mixed with 1:200,000 epinephrine). In the endonasal approach, the incision followed the caudal border of the lateral crus, cephalad to a soft triangle at the nostril apex; then immediately continued adjacent to the caudal border of the medial and middle crura along the columella rim; and extended along the caudal border of the footplate of the medial crus onto the floor of the nasal vestibule bilaterally. In the open approach, a stair step transcolumellar incision was added (Fig. 1).

In this study, we emphasize a couple of recent changes in our procedure since the publication of the prior article. First, we excised the dog-ear that appeared at the caudal end of the extended footplate incision due to the projected tip. Second, the undermining of the inferior columellar area was subcutaneously carried out wider than before to obtain more tip projection and further improve the columello-labial angle. In the previous study, undermining was done just below the incision, namely around the columello-labial junction. But after modification, we widened the area of

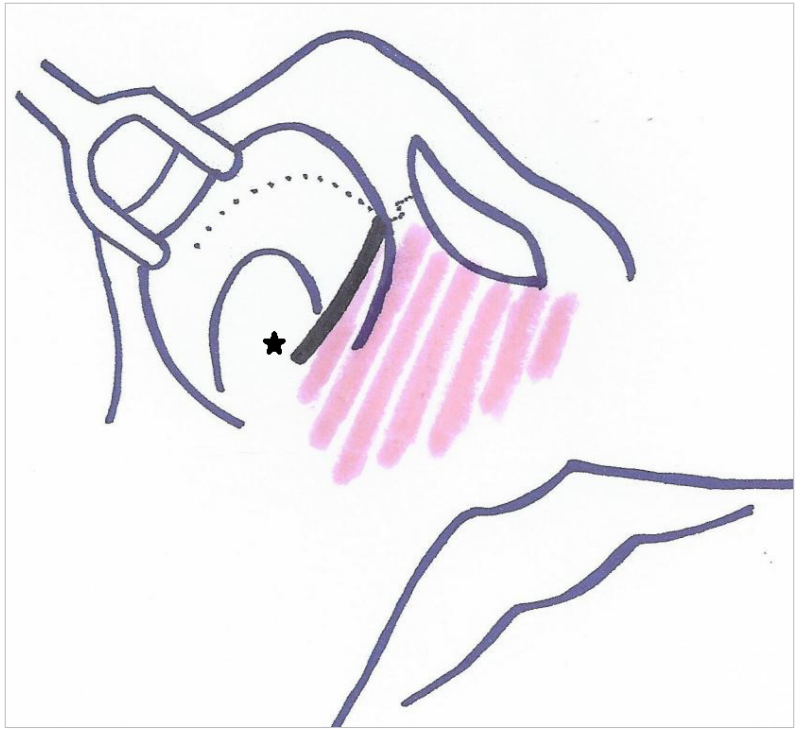

Fig. 1. The solid line represents the footplate incision and the star represents the dog-ear. And the modification of the area of undermining was also indicated. undermining to upper half of the upper lip. A pocket was then created in the nasal dorsum for implant insertion. A number of materials have been used for nasal augmentation, and the implant was fashioned and inserted into the dorsal subperiosteal pocket for dorsal augmentation and between the medial crura as the midcolumella strut.

This study included 85 additional patients ( 32 male and 53 female) who underwent aesthetic rhinoplasty using this extended footplate incision from August of 2010 to May of 2013. The mean age was 29 years and ranged from 20 to 59 years. Sixty of these patients underwent endonasal rhinoplasty (Fig. 2 and 3), while 25
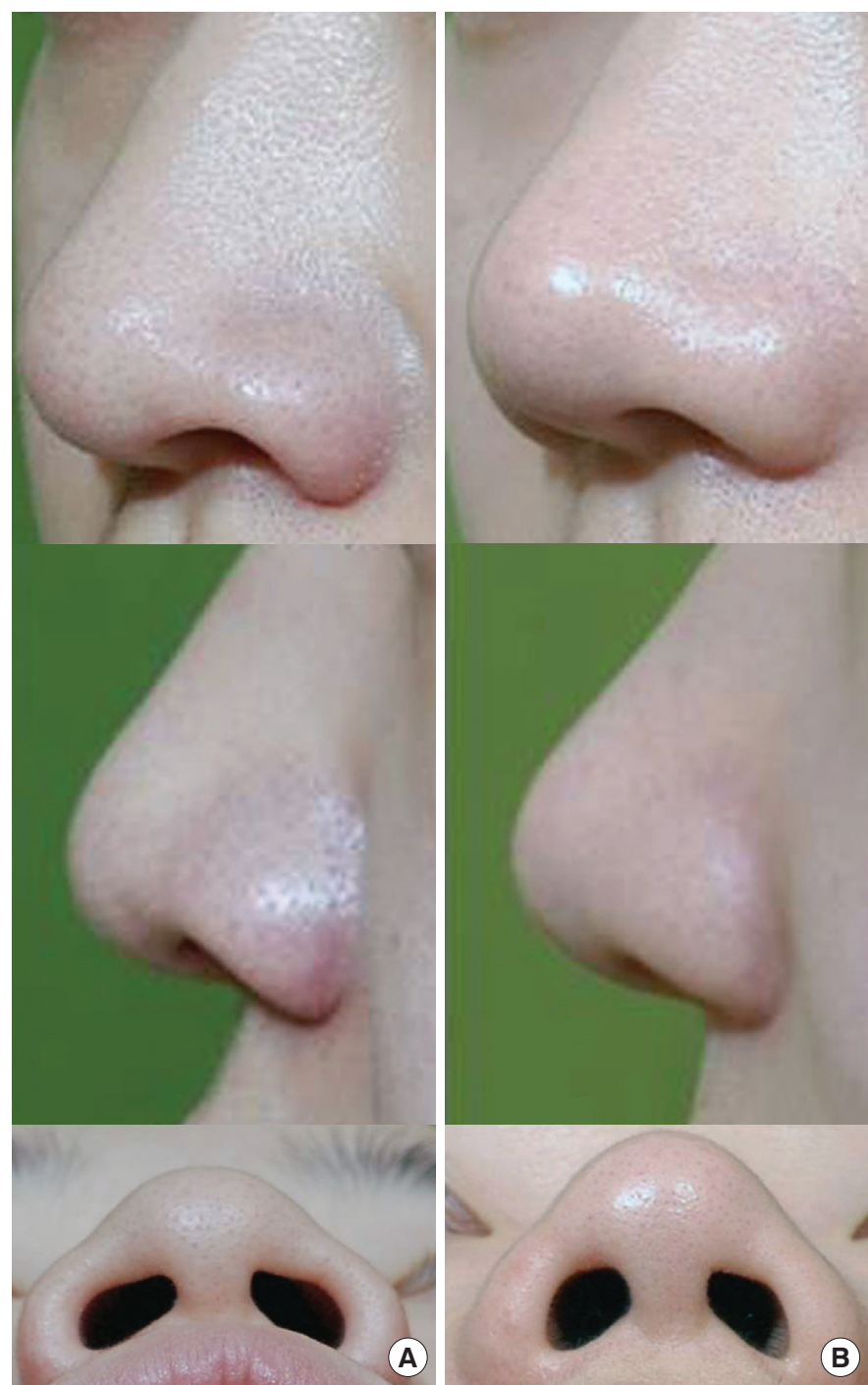

Fig. 2. (A) Preoperative appearance showing a poorly projected tip, an acute nasolabial angle and a wide alar base. This patient underwent resection of the depressor septi muscle and alar base augmentation through the footplate incision only. (B) Postoperative appearance at 13 months. Tip projection increased, nostril shape improved, and columello-labial angle also increased. 
patients underwent open rhinoplasty (Fig. 4). Seventy-one cases consisted of primary rhinoplasties while 14 had had previous nasal augmentations. Cartilage work was done in every open rhinoplasty case and cartilage onlay graft was added in 18 cases. The postoperative results were evaluated for at least 12 months following the procedure. A total of 43 patients had an adequate follow-up time, with an average of 15 months. Finally, patient satisfaction and postoper-

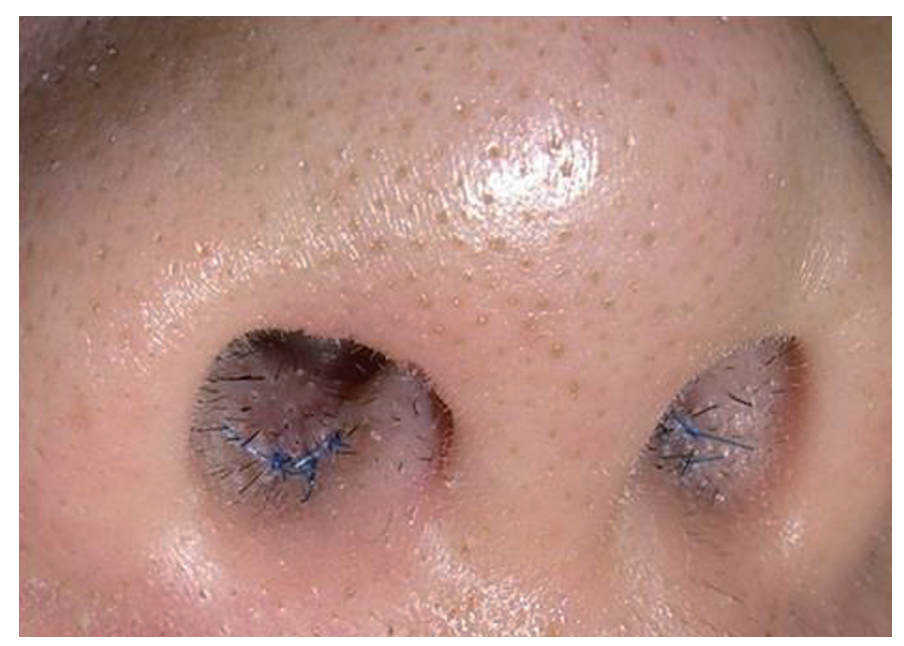

Fig. 3. Postoperative appearance at 2 days showing the suture site of the footplate incision. ative complications were recorded.

\section{RESULTS}

All patients showed an improvement in nasal height, tip projection, and nostril shape. The majority of the patients (40/43 cases; 96\%) were satisfied with their nasal shape, and the three dissatisfied patients wanted further nose augmentation. Interestingly, 30\% of the patients complained of an over rotated or over projected tip in the early postoperative period, but all of these patients became accustomed to and satisfied with their new nasal shape within 1 month of the procedure, even though the tip projection did not diminish. Two patients exhibited complications consisting of a mild nostril scar contracture, but this condition improved within 6 months postoperatively. Although a mild degree of asymmetry was noted in the nostril shapes of some patients, neither the patients nor the surgeons felt that it was clinically significant. There were no cases of implant extrusion, displacement, or infection. The extended footplate incision does not cause any problems because the scar is completely concealed. In addition, there were no respiratory complaints, and no revision procedures were performed.

\section{DISCUSSION}

In some surgical innovative ideas, major problems or complications
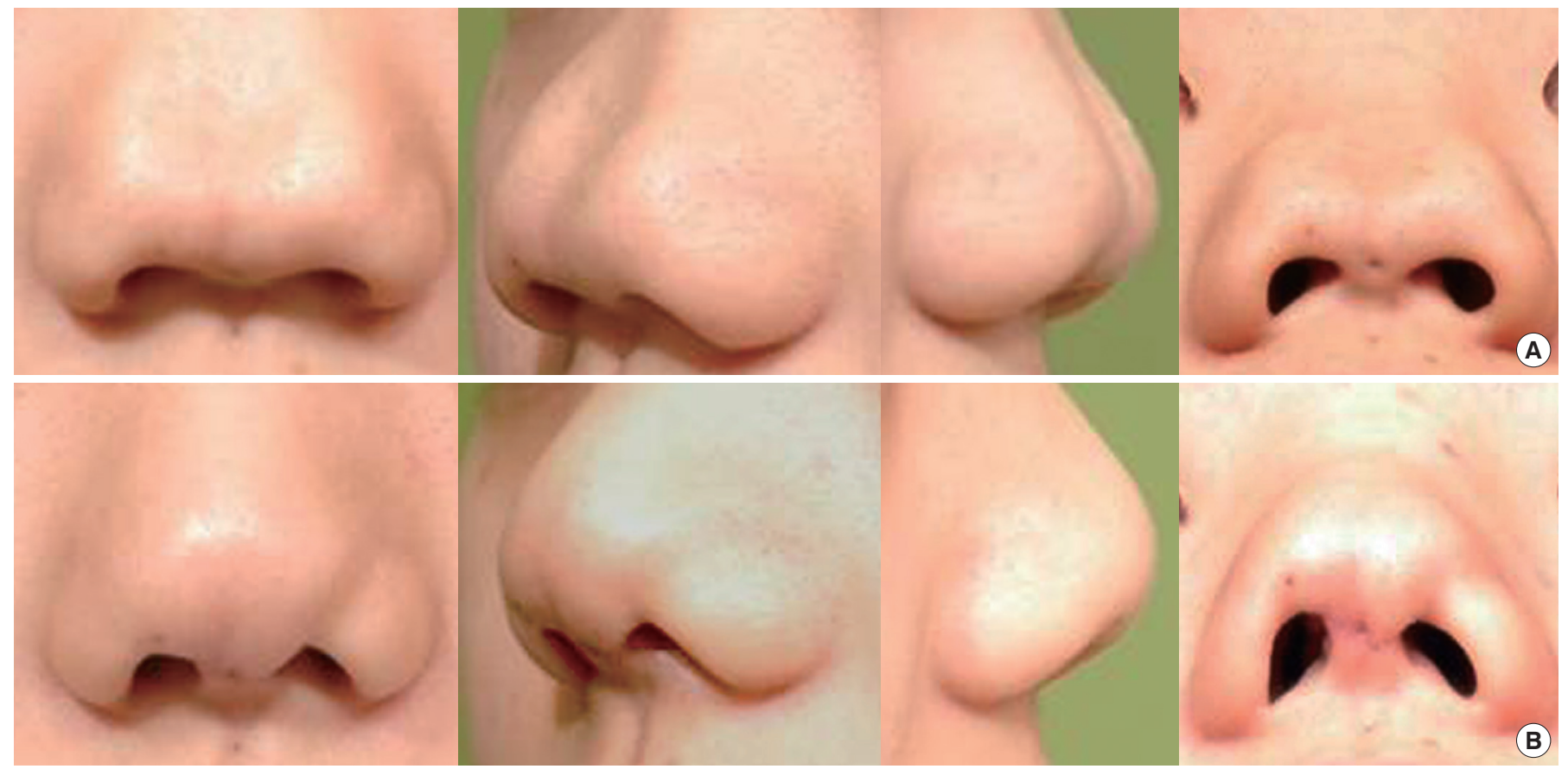

Fig. 4. (A) Preoperative appearance showing a low and wide nasal dorsum due to midfacial cleft, a depressed tip, and an acute nasolabial angle. This patient underwent dorsal augmentation with silicone, tip-plasty with a septal cartilage graft through the open approach with an extended footplate incision. (B) Postoperative appearance at 12 months. 
were found during the long term observation. These may result in abandonment of that methods after publication but future surgeons may still experience same problems if it is not updated. Therefore, the main focus of this article was to identify the safety and emphasize the efficiency of this footplate incision after long term followup. During the 10-year of long term follow-up and newly added cases since publication of the previous article, no significant complications were reported and we still think that this method contributes to Asian Rhinoplasty.

The anatomy of the footplates plays an important role in nasal tip support and in nasal base structure [6]. The position of the footplates of the medial crura determines the dimension, the shape, and the projection of those structures, and any modification of the footplates may compel a change in the overall scheme of the rhinoplasty. Moreover, the shape of the nostril and the base of the columella are controlled. In Asians, the length of the footplate segment is much longer than that in Caucasians [7], and when operating on an Asian patient, a surgeon may be able to use a longer footplate segment to more effectively recreate the lower vault of the nose. As a result of these facts, we previously searched for procedures that would enable us to easily access the footplates of the medial crura. We therefore modified our footplate incision in both endonasal and open rhinoplasty to achieve a higher degree of satisfaction for aesthetic rhinoplasty in Asian patients.

The lateral curves of the medial crural footplates can be approximated to narrow and lengthen the columella. The columella was also caudally advanced, and thus the shape of the nostrils could be elongated. By simultaneously resecting the depressor septi muscle, significant rotation was possible in the cephalad tip. In addition, septal cartilage could be easily harvested without additional intercartilaginous and transfixion incisions. These manipulations might also be performed through a conventional open approach to rhinoplasty without our footplate incision. However, this incision requires only 2 more stitches and makes all of these procedures become much easier. More importantly, this simple additional incision can also allow further tip projection due to the reduction in pressure from the skin flap on the tip. To illustrate, extensive tip augmentation may result in the new nasal tip tilting upward too much or the tip implant may push the underlying alar cartilage dome downward as a result of the overlying skin flap pressure. With our footplate incision, more tissue can be borrowed from the lower columallar flap to reduce the overall pressure on the nasal tip. After making the new tip, a dog ear can be seen at the posterior end of the footplate incision. This indicates that further tip projection is created by the footplate incision. In the past, we left this dog ear because it can be naturally improved. However, some patients complained about this invisible lesion. Therefore, we modified our procedure to excise the dog-ear that appeared at the caudal end of the extended footplate incision. Since there is a natural limit to the amount of tip heightening possible, and since even a few millimeters can be most helpful, we believe that the footplate incision is very useful. In addition, wide undermining of the inferior columellar area is subcutaneously carried out to further improve the tip projection and columello-labial angle. In the previous study, undermining was done just below the incision, namely around the columello-labial junction. But after modification, we widened the area of undermining to upper half of the upper lip. Because Asians have thicker nose skin and overlying skin flap pressure is limitation of tip augmentation, reducing tension of inferior columellar flap by wide undermining is considerably helpful. We have recently had more success in correcting tip deformities by using this incision.

The endonasal approach is appropriate for select patients due to the lower chance of complications and shorter procedure time. Open rhinoplasty with a transcolumellar incision is a type of surgery that can be more easily controlled. However, an extended nasal tip edema and columellar scar is some of the disadvantages of using this method. An endonasal approach with an extended footplate incision allows a patient to avoid these disadvantages and provides a more controlled procedure, in a manner similar to the open method.

Implant extrusion or translucency is significant complications that result from dorsal and tip augmentation with silicone $[3,8]$. These conditions are mostly due to the overcorrection or high pressure of the overlying skin $[9,10]$. These complications have not yet been experienced with our extended footplate incision procedure with a sufficient follow-up time.

One limitation of this study is that there is no data comparing this incision with a control group. Because the purpose of this article is not reporting the superiority of this method, but reporting the safety, efficiency and technical refinement, we did not compare the result with the control group.

Any patient with an undesirable shape of the tip, nostril, columella, and nasolabial angle that requires access to the footplate of the medial crus can be considered as a candidate for this new incision. In addition, our extended footplate incision may be helpful when patients require a higher degree of tip projection and rotation. This technique may therefore be considered for use in primary or secondary cases.

\section{CONCLUSION}

We think that the extended footplate incision is an excellent choice for Asian rhinoplasty, and we continue to perform this method. We are convinced that the several technical changes reported in the present study contribute to the improvement of the procedure and to final outcomes.

\section{PATIENT CONSENT}

Patients provided written consent for the use of their images. 


\section{REFERENCES}

1. Zingaro EA, Falces E. Aesthetic anatomy of the non-Caucasian nose. Clin Plast Surg 1987;14:749-65.

2. Byun JS, Kim KK. Correction of asian short nose with lower lateral cartilage repositioning and ear cartilage grafting. Plast Reconstr Surg Glob Open 2013;1:e45.

3. Jung DH, Moon HJ, Choi SH, et al. Secondary rhinoplasty of the Asian nose: correction of the contracted nose. Aesthetic Plast Surg 2004;28:1-7.

4. Park JH, Mangoba DC, Mun SJ, et al. Lengthening the short nose in Asians: key maneuvers and surgical results. JAMA Facial Plast Surg 2013;15:439-47.

5. Han SK, Ko HW, Kim WK. Advantages of adding a footplate incision in Asian rhinoplasty. Ann Plast Surg 2004;53:65-9.
6. Guyuron B. Footplates of the medial crura. Plast Reconstr Surg 1998; 101:1359-63.

7. Dhong ES, Han SK, Lee CH, et al. Anthropometric study of alar cartilage in Asians. Ann Plast Surg 2002;48:386-91.

8. Shirakabe Y, Shirakabe T, Takayanagi S. A new type of prosthesis for augmentation rhinoplasty: our experience in 1600 cases. Br J Plast Surg 1981;34:353-7.

9. Deva AK, Merten S, Chang L. Silicone in nasal augmentation rhinoplasty: a decade of clinical experience. Plast Reconstr Surg 1998;102: 1230-7.

10. Shirakabe Y, Shirakabe T, Kishimoto T. The classification of complications after augmentation rhinoplasty. Aesthetic Plast Surg 1985;9:18592. 